\title{
Система внутреннего аудита и контроля как элемент обеспечения экономической безопасности предприятия
}

\author{
Светлана Ракутько*, Елена Селезнева, Валерия Хафизова, \\ Валерия Страхова
}

Дальневосточный федеральный университет, г. Владивосток, Россия

\author{
Информация о статье \\ Поступила в редакичию: \\ 26.01.2020 \\ Принята \\ к опубликованию: \\ 13.05.2020 \\ УДК 338.3 \\ JEL M42, G30, M11
}

\begin{abstract}
Ключевые слова:
экономическая безопасность, внутренний аудит, внутренний контроль, риски, внешние угрозы, внутренние угрозы, служба внутреннего аудита и контроля
\end{abstract}

Keywords:

economic security, internal audit, internal control, risks, external threats, internal threats, internal audit and control services

\begin{abstract}
Аннотация
В работе предложены авторские подходы $\kappa$ понятиям «внутренний контроль» и «внутренний аудит», раскрыты их содержательные особенности, функиии, формы организации на предприятиях, определены ключевые задачи систем внутреннего аудита и внутреннего контроля в аспекте обеспечения экономической безопасности. Проанализированы возможные варианты взаимодействия систем внутреннего аудита и внутреннего контроля и предложили к использованию наиболее подходящую модель их комплексного взаимодействия для осуществления единовременного контроля финансово-хозяйственных прочессов и управления рисками компании.
\end{abstract}

The System of Internal Audit and Control as the Element to Ensure an Enterprise Economic Security

Svetlana Rakutko, Elena Selezneva, Valeriya Khafizova, Valeriya Strakhova

\begin{abstract}
The company economic security is the result of its effective financial and economic activities. This relationship is due to the pattern of economic development and increased competition between economic entities. The current market mechanisms significantly impact on the company activities, that in turn necessitates them to look for the ways to ensure the financial stability and economic security. This article aims at studying the role the internal audit and internal control play to ensure the company financial stability and the impact of these tools on its economic security. The paper describes the authors' approaches to the concepts of "internal control" and "internal audit", shows their content features, functions and forms of arrangement within a company. It also specifies the key objectives of the internal audit and internal control systems for ensuring the economic security.
\end{abstract}

* Автор для связи: rakutko.syu@dvfu.ru

DOI: https://dx.doi.org/10.24866/2311-2271/2020-1/55-64 
The authors analyzed the possible interactions of the internal audit and internal control systems and suggested the most appropriate model for their comprehensive interaction to be used for the simultaneous control of financial and economic processes and for the company risk management.

\section{Введение}

В условиях рыночной экономики предприятия подвержены влиянию негативных факторов (угроз), повторяющихся с неопределённой периодичностью и оказывающих отрицательное воздействие на финансовое состояние компаний. Неопределённость и турбулентность рыночной среды приводят к масштабным финансовым потерям у собственников компаний, инвесторов и государства. В таких условиях стабилизация хозяйственно-финансовой ситуации на предприятии путем своевременного определения потенциальных рисков с целью минимизации последствий влияния рыночных факторов на его деятельность является одним из приоритетных способов обеспечения экономической безопасности хозяйствующего субъекта.

Эффективное управление финансово-хозяйственной деятельностью предприятия базируется, в первую очередь, на использовании совокупности актуальных и достоверных данных о его финансовом положении, которое обеспечивается процедурами внутреннего аудита и контроля.

Вопросы применения внутреннего аудита и внутреннего контроля для отечественной науки являются относительно новыми. Отдельные аспекты в данном направления раскрыты такими российскими учеными, как Р.А. Алборов [1], В.Д. Андреев [2], И.А. Белобжецкий [3], А.М. Богомолов [4], В.В. Бурцев [5], С.И. Жминько [6], Г.В. Кулинина [7], Н.В. Лапухин [8], А.К. Макальская [9], Р.В. Макеев [10], С.В. Панкова [11], В.И. Подольский [12], В.Ю. Реутов и Ф.Б. Риполь-Сарагоси [13], В.А. Хмельницкий [14], А.Д. Шеремет [15] и др. Для толкования исследуемых понятий, определения их компетенций в системе управления и оценки их влияния на обеспечение экономической безопасности предприятия необходимо рассмотреть содержательные особенности внутреннего контроля и аудита, выявить разграничение их функций.

Цель исследования - проанализировать возможные варианты взаимодействия систем внутреннего аудита и внутреннего контроля и предложить способ их комплексного применения для единовременного контроля финансово-хозяйственных процессов и управления рисками.

\section{Теоретическая основа исследования}

Современная специальная литература по данному направлению отличается дискуссионным характером, и, как следствие, многообразием подходов к содержанию понятий «внутренний аудит» и «внутренний контроль». Некоторые авторы говорят о тождественности данных понятий, но большинство специалистов относят их к двум самостоятельным, но взаимодополняющим видам деятельности. Несмотря на то, что внутренний аудит и внутренний контроль обеспечивают достижения одной и той же цели - повышение эффективности финансово-хозяйственной деятельности предприятия, - приоритетные задачи у них разные. В связи с этим, при определении содержания 
рассматриваемых понятий, будем исходить из задач, стоящих перед внутренним контролем и внутренним аудитом.

Основными задачам внутреннего контроля многие авторы считают проверку наличия и сохранности имущества предприятия, соответствия хозяйственных операций действующему законодательству, достоверности финансовой отчётности; выявление отклонений и нарушений в работе предприятия [16].

К задачам внутреннего аудита относят проверку эффективности системы внутреннего контроля, разработку целостной системы управления рисками и анализ результатов её работы, а также разработку мероприятий по снижению рисков, контроль за соблюдением принципов корпоративного управления [6]. Таким образом, внутренний контроль направлен на решение повседневных задач по оперативному управлению и сбору информации о допущенных ошибках персоналом предприятия. Внутренний аудит охватывает широкий круг вопросов, связанных с предоставлением объективных независимых консультаций руководству предприятия, необходимых для принятия обоснованных управленческих решений по обеспечению финансовой стабильности.

В процессе исследования мы проанализировали существующие подходы к толкованию понятий «внутренний контроль» и «внутренний аудит» и пришли к выводу, что наиболее распространённым является подход, который рассматривает термин «внутренний контроль» как процесс. Сторонниками данного подхода являются С.И. Жминько [6], Ф.Б. Риполь-Сарагоси и В.Ю. Реутов [13], Р.В. Макеев [10] и др. По мнению ученых, внутренний контроль является процессом, который направлен на достижение целей и является результатом планирования, организации и мониторинга деятельности предприятия в целом и её отдельных структурных элементов.

Другой подход заключается в рассмотрении внутреннего контроля как системы наблюдения за деятельностью субъекта хозяйствования и проверки его фактического состояния $[2,7,14,15]$. Основная цель такой проверки оценка обоснованности и эффективности принятия управленческих решений и результатов их выполнения. Проверка позволяет выявить отклонения от поставленных задач и планируемых показателей, устранить негативные ситуации и при необходимости информировать об этом руководство предприятия.

Еще одним подходом к трактовке понятия «внутренний контроль» является рассмотрение внутреннего контроля как вида деятельности $[5,8$, 12]. Сторонники рассматривают внутренний контроль как независимую деятельность предприятия по проверке в интересах руководителя работы филиалов, цехов, участков, бригад, отделов, служб и других внутрихозяйственных подразделений. Данный вид контроля осуществляют штатные контролеры-ревизоры (аудиторы).

Большой энциклопедический словарь под редакцией А.М. Прохорова поясняет задачи внутреннего контроля по сбору информации о финансовом положении предприятия, акцентируя внимание на том, что обеспечить данный процесс может устойчивая и целенаправленная совокупность взаимосвязанных действий [17]. Следовательно, внутренний контроль - это система совокупности методик и процедур, принятых руководством в качестве средств эффективного управления деятельностью предприятия, а также опре- 
деленный вид деятельности различных служб по выявлению нарушений на предприятии.

Обобщая выше сказанное, под термином «внутренний контроль» предлагаем понимать эффективный инструмент управления деятельностью предприятия, усиленный функционирующей контрольной средой - деятельностью различных служб, направленной на выполнение контрольных процедур, базирующихся на утвержденных руководством методиках.

Проанализировать и оценить эффективность системы внутреннего контроля можно с помощью внутреннего аудита [18]. Профессиональный стандарт «Внутренний аудитор» регламентирует «независимые проверки и консультации по вопросам надежности и эффективности функционирования систем управления рисками, внутреннего контроля, корпоративного управления, операционной деятельности и информационных систем организации, с целью достижения стратегических целей организации...» [19].

Вместе с тем, многие авторы понимают внутренний аудит, как независимую экспертизу финансовой отчетности на основе проверки соблюдения порядка ведения бухгалтерского учета, соответствия хозяйственных и финансовых операций законодательству Российской Федерации, полноты и точности отражения в отчетности деятельности предприятия [1]. Известные экономисты В.В. Бурцев [5], А.К. Макальская [9], А.М. Богомолов [4], В.И. Подольский [12], С.В. Панкова [11] рассматривают этот термин как инструмент корпоративного управления рисками. В международном стандарте аудита №610 данное понятие также представлено как консультационная деятельность, целью которой является оценка и повышение действенности процессов корпоративного управления организацией, управления рисками, а также процессов внутреннего контроля [20].

Внутренний аудит и внутренний контроль, по мнению авторов, тесно взаимосвязаны и дополняют друг друга в вопросах применения различных процедур и решении задач. Основной причиной организации внутреннего аудита на предприятиях является обеспечение защиты от ошибок и злоупотреблений полномочиями сотрудниками предприятия, определение рисков хозяйственной деятельности и выявление слабых мест в системе управления компанией. С одной стороны, внутренний аудит позволяет оценить эффективность, полноту и соответствие законодательству реализации бизнеспроцессов, а с другой, - определить приоритетные направления их развития и оценить планируемые к реализации мероприятия по прогнозным результатам совершенствования, тем самым, обеспечивая финансовую, кадровую, правовую, информационную функции экономической безопасности.

Таким образом, на основе вышеизложенного мы предлагаем авторское определение понятия «внутренний аудит»: действенный инструмент управления предприятием в достижении его целей посредством независимой оценки всех сторон его деятельности.

\section{Методология исследования}

В процессе поиска и изучения причинно-следственных связей экономических явлений с целью анализа и выбора возможных вариантов взаимодействия систем внутреннего аудита и внутреннего контроля и обоснования 
рекомендации к использованию существующей модели их комплексного взаимодействия, авторы применили общенаучные подходы (системный, комплексный, логический). Методологическую основу настоящего исследования составили анализ и синтез; систематизация и классификация; моделирование и визуализация; индукция и дедукция; сравнение и обобщение.

\section{Обсуждение полученных результатов}

Существенным моментом в создании эффективной системы внутреннего аудита и контроля являются процедуры, методы, формы и субъекты контроля. В большинстве своем они являются общими как для внутреннего аудита, так и для внутреннего контроля. В современной практике приняты следующие формы организации этих систем: собственная служба внутреннего аудита и контроля, структурно-функциональный внутренний контроль (контроль осуществляется сотрудниками предприятия с привлечением внешних аудиторов для создания нормативной базы), ревизия (последующий (заключительный) контроль по распоряжению руководства предприятия), аутсорсинг (контроль осуществляется только с привлечением внешних консультантов), косорсинг (разделение контрольных функций между сотрудниками предприятия и внешними консультантами) [21].

Выбор форм организации системы внутреннего аудита и контроля зависит от сложности организационной структуры предприятия, правовой формы, целесообразности охвата контролем различных сторон деятельности, отношения руководства организации к контролю.

В условиях неопределенности особое внимание уделяется системам внутреннего аудита и контроля, которые агрегировали в себе не только традиционные процедуры контроля, но и интегрированы с системой оценки рисков в краткосрочной и долгосрочной перспективе. Такая система позволяет повысить уровень финансовой устойчивости и экономической безопасности предприятия.

В 2013 г. Международный институт внутренних аудиторов разработал модель «Линия трех защит», которая координирует процессы управления рисками и внутреннего контроля за счет четкого определения и разграничения функций и обязанностей. Основная причина появления такой модели связана с тем, что для создания эффективной системы управления рисками внутри организации необходимы три различные группы (линии защиты), которые действуют под руководством и контролем высшего менеджмента и Совета директоров. Каждая линия защиты обеспечивает выполнение определенных функций: первая линия защиты - управляет и контролирует риски, вторая - обеспечивает мониторинг рисков и осуществляет контроль, третья дает независимую оценку эффективности системы управления рисками. В итоге, если каждая линия защиты работает эффективно, возрастает вероятность того, что предприятие будет работать успешно в достижении своих целей [22]. Схематично данная модель представлена на рис.

Под первой линией защиты (бизнес-функции) подразумевается линейное руководство и самоконтроль работников, ответственных за оценку, регулирование и минимизацию рисков при выполнении хозяйственных операций. 


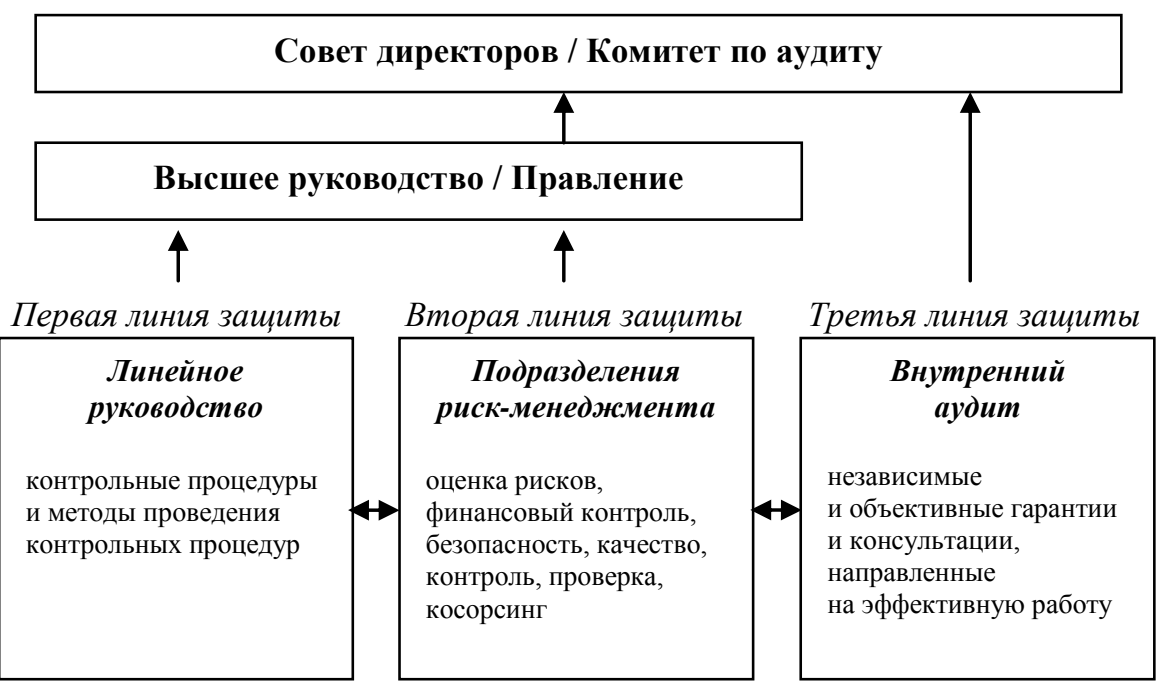

Рис. Модель «Линия трех защит»

Источник: составлено авторами на основе [21]

Для выполнения задач второй линии защиты (функции мониторинга) подключаются подразделения внутреннего контроля, обеспечивающие и отслеживающие внедрения эффективной практики управления рисками, внутреннего контроля, соблюдение законодательства, а также 19 административных правил и внутренних регламентов.

Третьей линией защиты (независимая функция) является внутренний аудит, информирующий высшее руководство предприятия об эффективности оценки рисков и управления ими, а также о качестве работы первой и второй линий защиты.

Для крупных предприятий существует еще четвертая линия защиты внешний аудит. Он обеспечивает внешнюю независимую оценку системы внутреннего аудита и контроля. С одной стороны, дает рекомендации руководству предприятия о выявленных нарушениях и недостатках в работе внутренних контролеров, а с другой, - обоснованное заключение пользователям о достоверности финансовой отчетности, которая гарантирует принятия эффективных решений о сотрудничестве.

Механизмом взаимодействия трех линий защиты является четкое и последовательное достижение поставленной цели. Каждая из трех линий защиты имеет одну и ту же цель - помощь предприятию в достижении эффективного функционирования. Все три линии служат одним и тем же заинтересованным лицам, имеют дело с одинаковыми проблемами риска и контроля. Для координации работы все этапы защиты должны обмениваться информацией о результатах проведенных контрольных процедур.

На первый взгляд данная модель может служить инструментом управления рисками только в крупных компаниях, так как к основным понятиям модели относятся «совет директоров», «высшее руководство», «комитет по аудиту». Но модель также может быть адаптирована для предприятий любого масштаба и вида деятельности. В каждом конкретном случае необходимо ис- 
ходить из ее целесообразности и ресурсоёмкости. Модель трёх линий защиты является доступным, эффективным механизмом, который координирует процессы управления рисками и внутреннего контроля за счет чёткого определения и разграничения соответствующих функций и обязанностей.

Данная модель направлена в первую очередь на более результативную работу системы внутреннего аудита и контроля за счёт выполнения следующих принципов:

- интеграции (обеспечивает связь службы внутреннего аудита и контроля с прочими структурными подразделениями предприятия, её влияние на их деятельность);

- комплексности (обеспечивает охват каждого структурного подразделения и отдела, бизнес-процесса);

- ответственности (предполагает ответственность каждого сотрудника, включённого в процесс осуществления внутреннего аудита и контроля, за выполнение соответствующих функций и задач);

- постоянства (определяет непрерывную контролирующую деятельность службы внутреннего аудита и контроля);

- разрешения и одобрения (подразумевает наличие формальных процедур санкционирования финансово-хозяйственных операций);

- распределения обязанностей (обеспечивает рациональное распределение задач между сотрудниками с целью их равномерной нагрузки и исключения злоупотреблений);

- сбалансированности (подразумевает обеспечение сотрудников службы техническими и программными инструментами для реализации контрольных функций);

- своевременности (создает возможность информирования о выявленных несоответствиях);

- соответствия (предполагает соотнесение службы внутреннего аудита и контроля с особенностями бизнес-процессов, с организационной структурой предприятия, её быстрой адаптацией к внутриорганизационным изменениям) [23].

Соблюдение данных принципов при создании и функционировании системы внутреннего аудита и контроля повысит её эффективность и укрепит доверие заинтересованных пользователей к информации о деятельности предприятия.

\section{Заключение}

В ходе исследования авторы проанализировали существующие подходы к определению понятий «внутренний контроль» и «внутренний аудит», сформулировали собственный авторский подход к их содержанию, исследовали механизмы взаимодействия внутреннего аудита и контроля и выявили комплексный характер их влияния на экономическую безопасность предприятия.

В исследовании представлена модель трёх линий защиты, которую авторы рекомендуют для включения в систему внутреннего аудита и контроля на предприятиях с различным масштабом деловой активности с условием адаптации данной модели к определенному виду деятельности компании и с 
учётом оценки целесообразности её применения. Внедрение данной модели позволит руководству оценить систему внутреннего аудита и контроля, финансовое состояние предприятия и определить перспективы его развития. Данная модель поможет менеджерам не только обеспечить действенный контроль над финансово-хозяйственными процессами за счёт разграничений обязанностей по выявлению и оценке рисков и контролю, но и избежать дублирования контрольных процедур различными службами предприятия.

В процессе дальнейших исследований авторы статьи планируют разработать способы адаптации модели для применения отдельными предприятиями региона.

\section{Список источников / References}

1. Алборов Р. А. Аудит в организациях промышленности, торговли и АПК: учебное пособие. Ижевск, Ижевская ГСХА, 2016. 284 с. [Alborov R. A. Audit v organizaciyax promy`shlennosti, torgovli i APK: uchebnoe posobie [Audit in organizations of industry, trade and agribusiness: a training manual]. Izhevsk, Izhevsk State Agricultural Academy Publ., 2016. 284 p.]

2. Андреев В.Д. Основы интегрированного риск-ориентированного внутреннего контроля и аудита хозяйствующих субъектов: учебное пособие. Москва, Магистр ИНФРА-М, 2019. 365 с. [Andreev V.D. Osnovy` integrirovannogo riskorientirovannogo vnutrennego kontrolya i audita xozyajstvuyushhix sub"ektov: uchebnoe posobie [Andreev V.D. Osnovy` integrirovannogo risk-orientirovannogo vnutrennego kontrolya i audita xozyajstvuyushhix sub“ektov: uchebnoe posobie]. Moscow, Master of INFRA-M Publ., 2019. 365 p.]

3. Белобжецкий И.А. Финансовый контроль и новый хозяйственный механизм. Москва, Финансы и статистика, 1989. 256 с. [Belobzheczkij I.A. Finansovy`j kontrol’ i novy j xozyajstvenny`j mexanizm [Financial control and a new economic mechanism]. Moscow, Finance and Statistics Publ., 1989. 256 p.]

4. Богомолов А.М. Внутренний аудит: словарь-справочник. Москва, изд-во ПРИOP, 2002. 330 c. [Bogomolov A.M. Vnutrennij audit: slovar`-spravochnik [Internal audit: reference dictionary]. Moscow, PRIOR Publ., 2002. 330 p.]

5. Бурцев В.В. Методология и организация внутреннего аудита. Бухгалтер и за$к о н, ~ 2006$, №9, сc. 37-43. [Burcev V.V. Metodologiya i organizaciya vnutrennego audita [Methodology and organization of internal audit]. Buxgalter $i$ zakon $=A c$ countant and Law, 2006, no. 9, pp. 37-43.]

6. Жминько С.И. Внутренний аудит. Москва, Феникс, 2017. 320 с. [Zhmin`ko S.I. Vnutrennij audit [Internal audit]. Moscow, Phoenix Publ., 2017. 320 p.]

7. Кулинина Г.В. Внутренний контроль в системе управления компанией. Транспортное дело России, 2014, №5, сc. 177-179. [Kulinina G.V. Vnutrennij kontrol’ v sisteme upravleniya kompaniej [Internal control in the company management system]. Transportnoe delo Rossii = Transport business in Russia, 2014, no. 5, pp. 177-179.]

8. Лапухин Н.В., Подольский В.Н. Экономическому контролю - современные технологические решения. Москва, Финансы и статистика, 1989. 118 с. [Lapuxin N.V., Podol`skij V.N. E`konomicheskomu kontrolyu - sovremenny`e texnologicheskie resheniya [Economic control - modern technological solutions]. Moscow, Finance and Statistics Publ., 1989. 118 p.]

9. Макальская А.К. Внутренний аудит: учебно-практическое пособие. Москва, изд-во «Дело и сервис». 2000. 80 с. [Makal`skaya A.K. Vnutrennij audit: uchebno- 
prakticheskoe posobie [Internal audit: a training manual]. Moscow, "Business and Service" Publ., 2000. 80 p.]

10. Макеев Р.В. Постановка систем внутреннего контроля: от проверки отчетности к эффективности бизнеса. Санкт-Петербург, Бейкер Тилли Русаудит, 2008. 287 c. [Makeev R.V. Postanovka sistem vnutrennego kontrolya: ot proverki otchetnosti k e`ffektivnosti biznesa [Setting up internal control systems: from reporting verification to business performance]. St. Petersburg, Baker Tilly Rusaudit Publ., 2008. 287 p.]

11. Панкова С.В. Международные стандарты аудита. Москва, Магистр, 2008. 288 c. [Pankova S.V. Mezhdunarodny`e standarty' audita [International Auditing Standards]. Moscow, Magister Publ., 2008. 288 p.]

12. Подольский В.И. Аудит. Москва, Юрайт, 2012. [Podol`skij V.I. Audit [Audit]. Moscow, Yurait Publ., 2012. Available at: http://alleng.org/d/econ-fin/econfin404.htm

13. Риполь-Сарагоси Ф.Б., Реутов В.Ю. Внутренний аудит: организация и планирование: учебное пособие. Ростов-на-Дону, Феникс, 2006. 189 с. [Ripol'-Saragosi F.B., Reutov V.Yu. Vnutrennij audit: organizaciya i planirovanie: uchebnoe posobie [Internal Audit: Organization and Planning: A Training Manual]. Rostov-on-Don, Phoenix Publ., 2006. 189 p.]

14. Хмельницкий В.А., Гринь Т.А. Контроль и аудит. Минск, Современная школа, 2006. [Xmel`niczkij V.A., Grin` T.A. Kontrol`i audit [Control and audit]. Minsk, Modern School Publ., 2006.

15. Шеремет А.Д., Суйц В.П. Аудит. Москва, ИНФРА-М, 7-е издание, 2019. 375 с. [Sheremet A.D., Sujcz V.P. Audit [Audit]. Moscow, INFRA-M Publ., 2019. 375 p.]

16. Казакова Н.А., Ефремова Е.И. Концепция внутреннего контроля эффективности организации. Москва, ИНФРА-М, 2015. 234 с. [Kazakova N.A., Efremova E.I. Koncepciya vnutrennego kontrolya e'ffektivnosti organizacii [Organization internal control concept]. Moscow, INFRA-M Publ., 2015. 234 p.]

17. Прохоров А.М. Большой энциклопедический словарь. Санкт-Петербург, Норинт, 2004. 1456 с. [Proxorov A.M. Bol`shoj e`nciklopedicheskij slovar` [Great Encyclopedic Dictionary]. St. Petersburg, Norint Publ., 2004. 1456 p.]

18. Басюк А.С. Система внутреннего контроля как элемент обеспечения экономической безопасности туристского предприятия. Научно-методический электронный журнал «Кониепт», 2016, №6, сс. 1-11. [Basyuk A.S. Sistema vnutrennego kontrolya kak e`lement obespecheniya e`konomicheskoj bezopasnosti turistskogo predpriyatiya [The internal control system as an element of ensuring the economic security of a tourist enterprise]. Nauchno-metodicheskij e lektronny j zhurnal "Koncept» = Scientific and methodological electronic journal "Concept", 2016, no. 6, pp. 1-11.]

19. Профессиональный стандарт «Внутренний аудитор», утв. Приказом Министерства труда и социальной защиты РФ от 24.06.2015 № 398н. [Professional’ny’j standart "Vnutrennij auditor», utv. Prikazom Ministerstva truda i social'noj zashhity" RF ot 24.06.2015 № 398n [The professional standard “Internal Auditor", approved by Order of the Ministry of Labor and Social Protection of the Russian Federation dated June 24, 2015 No. 398n]

20. Международный стандарт аудита № 610 «Использование работы внутренних аудиторов» введён в действие на территории Российской Федерации Приказом Минфина России от 24.10.2016 № 192н. [Mezhdunarodny`j standart audita № 610 «Ispol zovanie raboty' vnutrennix auditorov» vvedyon v dejstvie na territorii Rossijskoj Federacii Prikazom Minfina Rossii ot 24.10.2016 № 192n [The international audit standard No. 610 "Using the work of internal auditors" was put into effect 
on the territory of the Russian Federation by Order of the Ministry of Finance of Russia dated 24.10.2016 No. 192H].

21. Бабич А.А. Сиротина, Е.П. Формы организации внутреннего контроля на предприятии. Пенза, 2019. [Babich A.A. Sirotina, E.P. Formy` organizacii vnutrennego kontrolya na predpriyatii [Forms of organization of internal control at the enterprise]. Penza, 2019.]

22. Anderson J., Gina Eubanks. Effective use of COSO in three line of defense models. Available at: https://www.iia-ru.ru

23. Абдулаева И.И. Методические основы организации внутреннего контроля. Вопросы экономики и права, 2012, №6, сc.119-122. [Abdulaeva I.I. Metodicheskie osnovy` organizacii vnutrennego kontrolya [Methodological foundations of the organization of internal control]. Voprosy`e konomiki i prava = Economics and Law, 2012, no. 6, pp. 119-122.]

\section{Сведения об авторах / About authors}

Ракутько Светлана Юрьевна, кандидат экономических наук, доцент, доцент кафедры бухгалтерского учета, анализа и аудита Школы экономики и менеджмента, Дальневосточный федеральный университет. 690922 Россия, г. Владивосток, о-в Русский, кампус ДВФУ, корпус G. E-mail: rakutko.syu@dvfu.ru

Svetlana Yu. Rakutko, Candidate of Economic Sciences, Associate Professor, Associate Professor of the Department of Accounting, Analysis and Audit, School of Economics and Management, the Far Eastern Federal University. Bldg. G, FEFU campus, Russky Island, Vladivostok, Russia, 690922.

E-mail: rakutko.syu@dvfu.ru

Селезнева Елена Юрьевна, кандидат исторических наук, доцент, доцент кафедры бухгалтерского учета, анализа и аудита Школы экономики и менеджмента, Дальневосточный федеральный университет. 690922 Россия, г. Владивосток, о-в Русский, кампус ДВФУ, корпус G.

E-mail: selezneva.eyu@dvfu.ru

Elena Yu. Selezneva, Candidate of Historical Sciences, Associate Professor, Associate Professor of the Department of Accounting, Analysis and Audit, School of Economics and Management, the Far Eastern Federal University. Bldg. G, FEFU campus, Russky Island, Vladivostok, Russia, 690922.

E-mail: selezneva.eyu@dvfu.ru

Хафизова Валерия Михайловна, магистрант кафедры бухгалтерского учета, анализа и аудита Школы экономики и менеджмента, Дальневосточный федеральный университет. 690922 Россия, г. Владивосток, о-в Русский, кампус ДВФУ, корпус G. E-mail: valeriya.mikhaylovna@mail.ru

Valeria M. Khafizova, Master Student of the Department of Accounting, Analysis and Audit, School of Economics and Management, the Far Eastern Federal University. Bldg. G, FEFU campus, Russky Island, Vladivostok, Russia, 690922. E-mail: valeriya.mikhaylovna@mail.ru

Страхова Валерия Андреевна, магистрант кафедры бухгалтерского учета, анализа и аудита Школы экономики и менеджмента, Дальневосточный федеральный университет. 690922 Россия, г. Владивосток, о-в Русский, кампус ДВФУ, корпус G. E-mail: strakhova.va@students.dvfu.ru

Valeria A. Strakhova, Master Student of the Department of Accounting, Analysis and Audit, School of Economics and Management, the Far Eastern Federal University. Bldg. G, FEFU campus, Russky Island, Vladivostok, Russia, 690922. E-mail: strakhova.va@students.dvfu.ru

(C) Ракутько С.Ю., Селезнева Е.Ю., Хафизова В.М., Страхова В.А. (C) Rakutko S.Yu., Selezneva E.Yu., Khafizova V.M., Strakhova V.A. Адрес сайта в сети интернет: http://jem.dvfu.ru 